\title{
Extension of the Kuramoto model to encompass time variability in neuronal synchronization and brain dynamics
}

\author{
Spase Petkoski, Aneta Stefanovska \\ From Twentieth Annual Computational Neuroscience Meeting: CNS*2011 \\ Stockholm, Sweden. 23-28 July 2011
}

The Kuramoto model (KM) is extended to incorporate at a basic level one of the most fundamental properties of living systems - their inherent time-variability. In building the model, we encompass earlier generalizations of the KM that included time-varying parameters in a purely physical way $[1,2]$ together with a model introduced to describe changes in neuronal synchronization during anæsthesia [3], as one of the many experimentally confirmed phenomena $[4,5]$ which this model should address. We thus allow for the time-variabilities of both the oscillator natural frequencies and of the inter-oscillator couplings. The latter can be considered as describing in an intuitive way the non-autonomous character of the individual oscillators, each of which is subject to the influence of its neighbors. The couplings have been found to provide a convenient basis for modeling the depth of anæsthesia [3].

Non-autonomous natural frequencies in an ensemble of oscillators, on the other hand, have already been investigated and interpreted as attributable to external forcing [6]. Our numerical simulations have confirmed some interesting, and, at first sight counter-intuitive, dynamics of the model for this case, and have also revealed certain limitations of this approach. Hence, we further examine the other aspects of the frequencies' time-variability. In addition, we apply the Sakaguchi extension (see [3] and the references therein) of the original KM and investigate its influence on the system's synchronization. Furthermore, we propose the use of a bounded distribution for the natural frequencies of the oscillators. A truncated Lorentzian distribution appears to be a good choice in that it allows the Kuramoto transition to be solved analytically: the resultant expression for the mean field amplitude matches perfectly the results obtained numerically.

The work to be presented helps to describe time-varying neural synchronization as an inherent phenomenon of brain dynamics. It accounts for the experimental results reported earlier [4] and it extends and complements a previous attempt [3] at explanation.

Published: 18 July 2011

References

1. Rougemont J, Felix N: Collective synchronization in populations of globally coupled phase oscillators with drifting frequencies. Phys. Rev. E 2006, 73:011104.

2. Taylor D, Ott E, Restrepo JG: Spontaneous synchronization of coupled oscillator systems with frequency adaptation. Phys. Rev. E 2010, 81:046214.

3. Sheeba JH, Stefanovska A, McClintock PVE: Neuronal synchrony during anesthesia: A thalamocortical model. Biophys. J 95:2722-2727.

4. Musizza B, Stefanovska A, McClintock PVE, Palus M, Petrovcic J, Ribaric S, Bajrovic FF: Interactions between cardiac, respiratory and EEG- $\delta$ oscillations in rats during anæsthesia. J. Physiol 2007, 580:315326, Bahraminasab A, Ghasemi F, Stefanovska A, McClintock PVE, Friedrich R: Physics of brain dynamics: Fokker-Planck analysis reveals changes in EEG $\delta-\theta$ interactions in anæsthesia, New Journal of Physics 2009, 11: 103051.

5. Rudrauf $D$, et al: Frequency flows and the time-frequency dynamics of multivariate phase synchronization in brain signals. Neuroimage 2006, 31:209-227.

6. Choi MY, Kim YW, Hong DC: Periodic synchronization in a driven system of coupled oscillators. Phys. Rev. E 1994, 49:3825-3832.

doi:10.1186/1471-2202-12-S1-P313

Cite this article as: Petkoski and Stefanovska: Extension of the Kuramoto model to encompass time variability in neuronal synchronization and brain dynamics. BMC Neuroscience 2011 12(Suppl 1):P313.

* Correspondence: spase.petkoski@lancaster.ac.uk

Department of Physics, Lancaster University, Lancaster, LA1 4YB, UK 